\title{
Lidocaine in liposuction wetting solution has a minimal effect on analgesic requirements when used in conjunction with general anesthesia
}

DA Nickerson MD FRCSC ${ }^{1}$, PA Clugston MD FRCSC ${ }^{2}$, RJ Warren MD FRCSC ${ }^{2}$

DA Nickerson, PA Clugston, RJ Warren. Lidocaine in liposuction wetting solution has a minimal effect on analgesic requirements when used in conjunction with general anesthesia. Can J Plast Surg 2002;10(5):203-205.

Lidocaine has become a common additive to liposuction wetting solutions, even for liposuction conducted under general anesthesia. Recent focus on the morbidity associated with liposuction has prompted a review of this practice. The analgesic requirements of patients undergoing liposuction of multiple sites under general anesthetic, with and without a lidocaine-containing wetting solution, were reviewed. The results suggest that while lidocaine does provide a reduction in analgesic requirements, this reduction is small, is readily compensated for by oral analgesics, and may not balance the potential risks associated with lidocaine.

Key Words: Analgesia; General anesthesia; Lidocaine; Liposuction
La lidocaïne ajoutée à la solution d'infiltration utilisée en liposuccion sous anesthésie générale a un effet minimal sur les besoins en analgésiques

\footnotetext{
RÉSUMÉ : La lidocaïne est désormais souvent ajoutée aux solutions d'infiltration utilisées pour la liposuccion, même lorsque l'intervention est réalisée sous anesthésie générale. Un récent constat de la morbidité associée à la liposuccion a incité les spécialistes à examiner cette pratique. Ils ont évalué les besoins en analgésiques de patients subissant sous anesthésie générale des liposuccions en plusieurs zones, réalisées avec ou sans solution d'infiltration contenant de la lidocaïne. Les résultats donnent à penser que, bien que cette substance permette de réduire les besoins en analgésiques, cette réduction est faible et ne justifie pas les risques potentiels associés à la lidocaïne. De plus, ces besoins peuvent être facilement comblés par des analgésiques oraux.
}

Presented at the annual meeting of the Northwest Society of Plastic Surgeons, February 2000, Whistler Resort, British Columbia, Canada

${ }^{1}$ Division of Plastic Surgery, University of Calgary, Calgary, Alberta; ${ }^{2}$ Division of Plastic Surgery, University of British Columbia, Vancouver, British Columbia

Correspondence and reprints: Dr DA Nickerson, Suite 140, $14028^{\text {th }}$ Avenue Northwest, Calgary, Alberta T2N 1 B9.

Telephone 403-282-6800, fax 403-282-6890, e-mail drnickerson@telusplanet.net 
$\mathrm{T}_{\mathrm{i}}^{\mathrm{h}}$ he use of lidocaine in liposuction wetting solutions was initially pioneered by those seeking to perform liposuction under a combination of local anesthetic and sedation (or local anesthetic alone) as opposed to general anesthetic.

Lidocaine has since become an accepted constituent of wetting solutions for liposuction performed under general anesthesia. Proponents of the use of lidocaine in wetting solution cite analgesic benefits lasting up to $18 \mathrm{~h}$ postoperatively $(1,2)$. However, Perry et al (3) disputed the benefits of lidocaine in a small study of paired liposuction sites using patients as their own internal controls. The recent census survey by Grazer and de Jong (4) revealed 130 deaths following liposuction between 1994 and 1998. In that and other studies, lidocaine toxicity was implicated as a potential factor in perioperative liposuction deaths, along with poor patient selection, excessive fluid resuscitation, pulmonary embolism and inadequate fluid resuscitation $(4,5)$. The work of Grazer and de Jong is arguably the most extensive compilation of liposuction deaths to date. In the absence of objective toxicology data, they could not definitively attribute any deaths to lidocaine toxicity. However, $28.5 \%$ of deaths in that survey fell into the "unknown or confidential' category.

Whether lidocaine toxicity accounts for a share of that $28.5 \%$ of liposuction deaths, the possibility of toxicity makes it incumbent upon plastic surgeons to be able to rationalize their use of lidocaine in the wetting solution of those patients having liposuction performed under general anesthetic.

To further examine the role of lidocaine in the wetting solution for liposuction of multiple sites performed under general anesthesia, the experience of two surgeons (one who uses a lidocaine-containing wetting solution and one who does not) were reviewed.

\section{METHODS}

This retrospective review was conducted in a free-standing aesthetic surgery facility. At the time of the study, two plastic surgeons worked with a single anesthesiologist. One surgeon's usual practice was to use a wetting solution containing lidocaine (Abbott Laboratories, USA). The other surgeon's usual practice was to use a lidocaine-free wetting solution.

The operating room and recovery room records of 59 consecutive American Society of Anesthesiologists Class I patients undergoing liposuction of multiple sites were reviewed. All patients received a standard general anesthetic protocol consisting of a weight-appropriate induction dose of Propofol (Abbott Laboratories, USA) and Fentanyl Citrate (Abbott Laboratories, USA). Additional intravenous Fentanyl Citrate was given intraoperatively in patients showing tachycardia, hypertension or signs of surgical awareness.

Twenty-eight of the patients ('Without Lidocaine' group) had their procedures done using tumescent fluid without lidocaine (Ringer's Lactate with $1 \mathrm{~mL} / \mathrm{L}$ of 1:1000 adrenaline) and 31 patients ('With Lidocaine' group) had liposuction using tumescent fluid with lidocaine (Ringer's Lactate with $25 \mathrm{~mL} / \mathrm{L}$ of $0.5 \%$ lidocaine with 1:200,000 adrenaline). The two groups were then compared using the following parameters: areas treated, wetting solution volume, liposuction aspirate volume, length of operative procedure, length of recovery room stay, narcotic utilization intra- and postoperatively, and complications.

Narcotic equivalencies with Morphine Sulfate (Abbott Laboratories, USA) as the standard (6) were used to determine total intraoperative and postoperative narcotic usage, according to the following values:

- $100 \mu \mathrm{g}$ of fentanyl citrate $=10 \mathrm{mg}$ of morphine sulfate

- $200 \mathrm{mg}$ of codeine $=10 \mathrm{mg}$ of morphine sulfate

- $75 \mathrm{mg}$ of meperidine $=10 \mathrm{mg}$ of morphine sulfate

On this basis, when comparing the common narcotic doses administered to the study patients, $100 \mu \mathrm{g}$ of fentanyl citrate is equal to 1 narcotic equivalent unit, one tablet of Tylenol \#3 (30 mg of codeine - Janssen-Ortho Pharmaceuticals, USA) is equal to 0.15 narcotic equivalent units, and $10 \mathrm{mg}$ of meperidine is equal to 0.13 narcotic equivalent units.

The two-sample, two-tailed t-test was used to test for difference between group means for each variable examined.

\section{RESULTS}

The two groups were similar in age and sex composition (Table 1). The areas treated with liposuction are shown in Table 2. The mean amount of tumescent fluid infiltrated was not significantly different between groups ('Without Lidocaine': 3.1 L versus 'With Lidocaine': 3.6 L; P>0.05). The mean amount of liposuction aspirate was not significantly different between groups ('Without Lidocaine': $2.4 \mathrm{~L}$ versus 'With Lidocaine': $3.5 \mathrm{~L}$; P>0.05). The operative times were not significantly different between groups ('Without Lidocaine': 125 min versus 'With Lidocaine': 131 min; $\mathrm{P}>0.05$ ).

Narcotic requirements were significantly higher for the 'Without Lidocaine' group both intraoperatively $(\mathrm{P}=0.009)$ and postoperatively $(\mathrm{P}=0.00015)$ (Table 3$)$.

There was no significant difference in length of recovery room stay between the two groups ('Without Lidocaine': 207 min versus 'With Lidocaine': 215 min). There were no perioperative complications in either group.

Cost analysis of the anesthetic drug cost (including all narcotics and lidocaine) showed a cost per case that was significantly less $(\mathrm{P}=0.0005)$ in the 'Without Lidocaine' group (\$1.27) versus the 'With Lidocaine' group (\$3.98).

\section{DISCUSSION}

Perry et al (3) contended that lidocaine provides no benefit in a small internal control study, and cited this as rationale for not using lidocaine as a wetting solution additive, to 
TABLE 1

Demographics of the two study groups

\begin{tabular}{ll}
\hline 'Without Lidocaine' group & 'With Lidocaine' group \\
\hline 28 patients & 31 patients \\
24 women, 4 men & All women \\
Mean age 39.7 years & Mean age 39.3 years \\
Age range $24-67$ years & Age range $23-52$ years \\
\hline
\end{tabular}

completely avoid potential toxicity.

The present study demonstrates a modest benefit from lidocaine, as measured by the amount of analgesic administered. The difference, however, was small. When one examines narcotic equivalencies, the average patient in the 'Without Lidocaine' group received the equivalent of only two additional tablets of Tylenol \#3. Because the intensity of postoperative pain following liposuction is typically minimal, management of the pain with mild oral analgesics is usually accomplished easily in most patients, with little or no risk.

As such, despite being at odds with Perry et al's conclusion that lidocaine provides no pain relief benefit for liposuction performed under general anesthesia, the present results do call into question whether lidocaine is of any practical benefit.

It has been demonstrated by other authors that peak serum levels of lidocaine are not reached for 9 to $14 \mathrm{~h} \mathrm{(1).}$ This profile creates the potential for complications because the patients at risk for lidocaine toxicity have typically long since been discharged and are at home without medical supervision.

Furthermore, because general anesthesia can mask the early signs, the first manifestation of lidocaine toxicity may be that of cardiovascular collapse.

In light of these issues, as well as Grazer and de Jong's recent calculated liposuction mortality rate of 19.1 per 100,000 (4), it is worthwhile to re-examine the role of the

\section{REFERENCES}

1. Klein JA. Tumescent technique for liposuction permits lidocaine doses of $35 \mathrm{mg} / \mathrm{kg}$ for liposuction. J Dermatol Surg Oncol 1990;16:248-63.

2. Rohrich RJ, Beran SJ, Fodor PB. The role of subcutaneous infiltration in suction-assisted lipoplasty: A review. Plast Reconstr Surg 1997;99:514-9.

3. Perry AW, Petti C, Rankin M. Lidocaine is not necessary in liposuction. Plast Reconstr Surg 1999;104:1900-2.
TABLE 2

Percentage of patients in each group undergoing liposuction of a given area

\begin{tabular}{lc}
\hline Area & Percentage \\
\hline 'Without Lidocaine' group & 32.1 \\
Hips, thighs and/or knees & 39.3 \\
Trunk & 25.0 \\
Trunk and hips, thighs and/or knees & 3.6 \\
Arms & \\
'With Lidocaine' group & 41.9 \\
Hips, thighs, and/or knees & 19.4 \\
Trunk & 35.5 \\
Trunk and hips, thighs, and/or knees & 3.2 \\
Arms & \\
\hline
\end{tabular}

TABLE 3

Intraoperative and postoperative narcotic requirements of the two study groups

\begin{tabular}{ll}
\hline Narcotic & Required dose \\
\hline 'Without Lidocaine' group & \\
Intraoperative Fentanyl & $249 \mu \mathrm{g}$ \\
Postoperative equivalents & 0.59 \\
'With Lidocaine' group & \\
Intraoperative Fentanyl & $191 \mu \mathrm{g}$ \\
Postoperative equivalents & 0.31 \\
\hline
\end{tabular}

routine use of lidocaine in liposuction performed under general anesthesia. Grazer and de Jong's inability to attribute a cause to $28.5 \%$ of the liposuction deaths in their survey underscores the importance of critical appraisal of any practices that could contribute to liposuction mortality.

Eliminating the practice of the routine addition of lidocaine to the wetting solution eliminates the possibility of lidocaine toxicity, at the expense of only a small increase in oral analgesic requirements.

4. Grazer FM, de Jong RH. Fatal outcomes from liposuction: Census survey of cosmetic surgeons. Plast Reconstr Surg 2000;105:436.

5. Rao RB, Ely SF, Hoffman RS. Deaths related to liposuction. N Engl J Med 1999;340:1471-5.

6. Welbanks L. Canadian Pharmacists Association Compendium of Pharmaceuticals and Specialties, 35th edn. Toronto: Webcom Limited, 2000. 